\title{
Natura: Towards Conversational Analytics for Comparing and Contrasting Time Series
}

\author{
Shima Imani \\ University of California, Riverside \\ siman003@ucr.edu
}

\author{
Eamonn Keogh \\ University of California, Riverside \\ eamonn@cs.ucr.edu
}

\begin{abstract}
Comparing and contrasting two things is one of the most basic ideas in science. While existing tools allow such comparisons for pairs (e.g. texts, trees, graphs, histograms), there are currently no such tools for time series data. This is somewhat surprising, given the ubiquity of time series data in modern life. One could imagine a tool for comparing and contrasting two time series by reporting various summary statistics. However, scientists, engineers, and physicians typically communicate such findings in natural language. In this work we present Natura, a domain agnostic natural language framework for comparing and contrasting two time series, that aims to duplicate this human skill. Through case study, we demonstrate the effectiveness and utility of our framework.
\end{abstract}

\section{KEYWORDS}

Time series; Conversational analytics; Contrasting time series; Natural language

ACM Reference Format:

Shima Imani and Eamonn Keogh. 2020. Natura: Towards Conversational Analytics for Comparing and Contrasting Time Series . In Companion Proceedings of the Web Conference 2020 (WWW'20 Companion), April 20-24, 2020, Taipei, Taiwan. ACM, New York, NY, USA, 2 pages. https://doi.org/10. $1145 / 3366424.3382690$

\section{INTRODUCTION}

Comparison of two objects is one of the most fundamental and fruitful tools in the history of science, however, to the best of our knowledge, there are essentially no tools for comparing and contrasting two time series. Given any experience observing practitioners in science, medicine or industry, their communications tend to be peppered with phrases comparing and contrasting pairs of time series. As an example, comparing the before-and-after sections of a single time series: "... after the percutaneous coronary intervention we noted the spikes in the ECG decreased" or, comparing two distinct time series of cooling towers "the chiller cycle time seems faster in the north tower than the south tower".

There are a handful of tools for comparing time series, most work by embedding time series into trees [5] or histograms, and then highlighting the differences in that space. While this can be fruitful, as we hinted above, it is somewhat removed from how practitioners do it.

This paper is published under the Creative Commons Attribution 4.0 International (CC-BY 4.0) license. Authors reserve their rights to disseminate the work on their personal and corporate Web sites with the appropriate attribution.

WWW'20 Companion, April 20-24, 2020, Taipei, Taiwan

(c) 2020 IW3C2 (International World Wide Web Conference Committee), published under Creative Commons CC-BY 4.0 License.

ACM ISBN 978-1-4503-7024-0/20/04

https://doi.org/10.1145/3366424.3382690
In this work, we introduce Natura (pronounced as ' $n a C H U r a$ '), a framework for comparing and contrasting time series using natural language. We organize the rest of the paper as follows: in Section 2 , we introduce the necessary notation and definitions. Section 3 introduces the vocabulary for comparing and contrasting time series data. We explain our framework Natura, in Section 4. Section 5 reviews related work. In Section 6, we perform an empirical evaluation. Section 7 draws conclusions and suggests directions for future work.

\section{DEFINITIONS AND NOTATION}

We begin by describing the necessary definitions and notation. The data type of interest is time series:

Definition 1 (Time series): A time series $T$ of length $n$ is a sequence of real-valued numbers: $t_{i}: T=t_{1}, t_{2}, \ldots, t_{n}$.

A local region of time series is called a subsequence:

Definition 2 (Subsequence): A subsequence $T_{i, m}$ of a time series $T$ is a continuous ordered subset of the values from $T$ of length $m$ starting from position $i . T_{i, m}=t_{i}, t_{i+1}, \ldots, t_{i+m-1}$, where $1 \leq i \leq n-m+1$.

Definition 3 (Time Series Corpus): Time series corpus is a large set of time series, which we use to perform statistical analysis, as we show in Section 4.

\section{VOCABULARY}

A fundamental assumption of our proposed method is that for any given subsequence, we can measure to what degree it can be said to possess the various features that a human might use to describe it, such as "smooth" or "symmetric". This is not a completely solved problem, but we will exploit recent progress in this direction [3]. We list and briefly explain a sample of words which consist of adjectives, comparative adjectives, adverbs, and conjunctions from our vocabulary. A small smaple of our adjectives are:

- rising (falling): The degree to which the slope of the best fit line is positive.

- symmetric (asymmetric): Rewards a subsequence for having an axis symmetry about its midpoint.

- spiky (dropout): Rewards a subsequence for having a small (but non-zero) number of data points much greater (less) than the mean.

- regular (irregular): Rewards a subsequence for being possibly repeated.

We need to be able to communicate the differences, using the following comparative adjectives: \{less, more, equal\}

We would like to be able to communicate the magnitude of the differences, thus we use the following adverbs:

\{slightly, moderately, considerably\} 
Finally, a simple list of sentences would feel very unnatural and disjointed. We can fix this with some simple conjunctions:

$$
\text { \{however, and }
$$

\section{NATURA}

We are now in a position to define the problem at hand.

Problem Definition: Given two time series, predict the sentences that most users would use to compare/contrast them.

We assume that the users are limited to the vocabulary and (informal) grammar discussed in the previous section. To generate a text summary, Natura consists of the following steps:

- Mapping time series to an adjective:

- For each adjective in our vocabulary, we define a function that takes a time series and subsequence length and outputs a meta-time series.

-We generate a histogram for the meta-time series we created in the first step. Each histogram corresponds to a word in our vocabulary.

- Generating comparative adjectives:

-We compute the difference between two time series histograms for each word using their differences. If the difference is negative, positive, or zero, we label it as less, more, and equal respectively.

- Generating adverbs:

- We use time series corpus (Definition 3) to generate an adverb for each adjective.

- We compute the distance between histograms for each adjective of time series in our corpus using their L2 norm. This will generate $2 n$ distances where $n$ is the number of time series in the corpus.

- We use standard deviation to rank our adverbs to s light ly, moderately and significantly.

- Generating conversation:

○ Using the comparative adjective and adverb that we generated for each adjective, we can create a sentence between two time series. For example, Natura might echo:

"time series A is slightly more spiky than time series B."

-We sort these sentences based on the adverb ranking and report them to the user.

- By default Natura reports the first two sentences. Natura uses "and" for conjunction of two sentences.

- If the comparative adjective in of the second sentence is less, then Natura uses "however" for the conjunction and changes the comparative adjective to more, and thus would change the order of time series.

\section{RELATED WORK}

There is a rich literature on contrasting and comparing graphs [6]. The pattern that differentiates between two graphs is called a contrast subgraph. The contrast subgraph is the smallest graph that appears in one graph but not in another graph dataset. Contrast subgraphs are often used to build graph classification models and high-level summaries.

In biology there is significant interest in contrasting protein and DNA sequences. Biologists are interested in finding the most significant subsequences within the protein sequences which discriminate between two sequences of protein [4]. One way to find these contrasting patterns is to search for a subsequence that is frequently observed in one of the sequences and not the other [1].

\section{EXPERIMENTAL EVALUATION}

An electrocardiogram ECG is telemetry that records electrical activity of the heartbeat. Figure 1 shows ten seconds of ECG data for two time series [2]. An attending physician might be interested in comparing the two following time series.

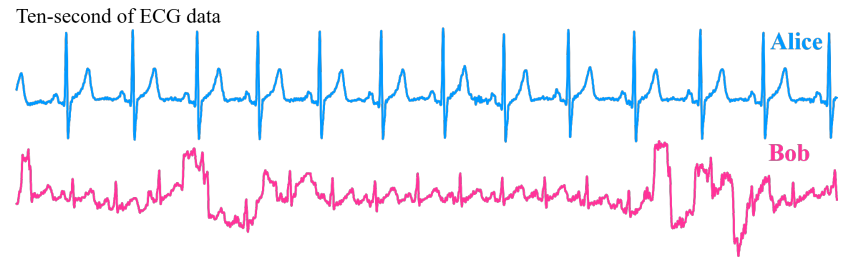

Figure 1: Time series of Alice and Bob corresponding to ECG data.

He may ask Natura for a comparison. Natura echos the most contrasting difference between two time series as:

Time series Alice is significantly more symmetric than time series $B o b$, however time series Bob is moderately more irregular than time series Alice.

Visually, we can verify that the outputs of Natura are reasonable. In this case, the existence of some artifacts in time series Bob makes this time series less symmetric and less regular.

\section{DISCUSSION AND CONCLUSIONS}

We have introduced Natura, a framework for comparing and contrasting time series data. One limitation of Natura is using the limited vocabulary introduced in Section 3. Therefore we intend to expand our vocabulary by adding more words from different fields including economics, statistics, manufacturing, and biology. We have deliberately kept Natura domain agnostic; however it may be useful to customize it for some domains. Finally, because Natura is a text-based comparison, we believe that it can leverage a wealth of information retrieval tools such as relevance feedback.

\section{REFERENCES}

[1] Guozhu Dong and James Bailey. 2012. Contrast data mining: concepts, algorithms, and applications. CRC Press.

[2] Ary L. Goldberger, Luis A. N. Amaral, Leon Glass, Jeffrey M. Hausdorff, Plamen Ch. Ivanov, Roger G. Mark, Joseph E. Mietus, George B. Moody, Chung-Kang Peng, and H. Eugene Stanley. 2000. PhysioBank, PhysioToolkit, and PhysioNet: Components of a New Research Resource for Complex Physiologic Signals. Circulation 101, 23 (June 2000), e215-e220. https://doi.org/10.1161/01.CIR.101.23.e215

[3] Shima Imani, Sara Alaee, and Eamonn Keogh. 2019. Putting the Human in the Time Series Analytics Loop. In Companion Proceedings of The 2019 World Wide Web Conference. ACM, 635-644.

[4] Steven G Johnson and R Stephen Howard. 2007. Contrasting patterns of synonymous and nonsynonymous sequence evolution in asexual and sexual freshwater snail lineages. Evolution: International Journal of Organic Evolution 61, 11 (2007), $2728-2735$.

[5] Jessica Lin, Eamonn Keogh, Stefano Lonardi, Jeffrey P Lankford, and Donna M Nystrom. 2004. Visually mining and monitoring massive time series. In Proceedings of the tenth ACM SIGKDD international conference on Knowledge discovery and data mining. ACM, 460-469.

[6] Roger Ming Hieng Ting and James Bailey. 2006. Mining minimal contrast subgraph patterns. In Proceedings of the 2006 SIAM International Conference on Data Mining. SIAM, 639-643. 